\title{
Design of Enhanced Differential Chaos Shift Keying Scheme
}

\author{
Nizar N. Al-Bassam
}

\begin{abstract}
In this paper, a new noncoherent Differential Chaos Shift Keying is proposed and named Extended -Differential Chaos Shift Keying (E-DCSK). Each pair of bits is modulated using two different versions of the same chaotic reference signal; the first information bit is modulated with the chaotic reference signal block while the next information bit is modulated after reversing samples' order of the same reference block. This saves the transmitted signal energy in addition to reduce the repeated components in the signal spectrum. Bit-error performance in additive-white-Gaussian-noise of the proposed system is analyzed and compared with the other chaos based communication systems. Results show that the bit-error rate performanceof the E-DCSK is often better than other schemes when spreading factorisrelatively low. Also, clear matching between theoretical analysis and simulation result is observed.
\end{abstract}

Index Terms-Noncoherent, differential chaos, spreading factor, spread spectrum.

\section{INTRODUCTION}

A spread spectrum technique has become one of the most attractive techniques for data transmission. It makes use of spreading signals bandwidth to carry information with low data rate utilizing the advantage of such technique like low probability of interception, resistance to jamming, and multiple access capabilityin addition to the immunity against multipath fading. Recently, Chaotic spread spectrum modulation techniques have been under focus of many researchers.It is based on modulating the information bits with chaotic samples generated from the specified chaotic map rather than conventional spreading functions. The chaotic samples have the properties of being widebandwidth, non-periodic, impulse like autocorrelation and relatively low cross correlation.

Generally, chaotic modulation systems can be divided into coherent and noncoherent [1]. In a coherent system, there is a need for the chaotic basis function to be available at the receiver while in noncoherent system, the existence of replica of the chaotic sequence in the receiver is not necessary for the detection process. Most of the proposed non-coherent systems use a reference signal method to despread the sequences at the receiver [2]-[8]. In DCSK, every single bit is represented by two similar chaotic sample functions. First sample function is used as a reference signal while the second sample function is used as information bearing signal. At the receiver, the received signal and its delayed version ismultiplied and averaged over the duration of the one chaotic

Manuscript received August 10, 2013; revised November 2, 2013.

Nizar N. Al-Bassam is with Department of Electronics and Communications, MEC, Oman (e-mail: Nazarhooby@yahoo.co.uk). sample function, thus, data rate is reduced to the half. To overcome the bandwidth problem of the DCSK, a Correlation Delay Shift Keying (CDSK) was suggested. In this system, the transmitted signal is the sum of the chaotic signal block and delayed version of the chaotic signal multiplied by the information bit, the system has less noise performance than DCSK, although data rate is increased. An enhanced version of DCSK is introduced in [3] by using same reference signal for multiple bits. System performance is better than CDSK and DCSK, but sending same chaotic sample function multiple times with the same duration $T_{c}$ leads to increase in the frequency components of $1 / T_{c}$ in the transmitted signal spectrum compared with the conventional DCSK. Permutation based-DCSK is used to build M-ary scheme in [4]. Security enhancement by destroying the similarity between reference signal samples and information bearing samples is suggested and implemented in [5]. Both systems also require sending two chaotic sample functions for each transmitted bit. A High efficiency DCSK is presented in [6]. Here, the information bearing signal part will be sum of two information bits modulated with two different chaotic reference signals. This doubles data rates but yields into much intrasignal interference which affects the BER destructively at low spreading factor. Another design for highly efficient DCSK is called code-shifted DCSK (CS-DCSK) [7]. The scheme uses Walsh code to separate the chaotic samples instead of using RF delay line. Again, more complicated systems is required. In this paper, a new scheme of DCSK is suggested. The system uses the same reference signal twice in different versions, which reduces the energy per bit compared with conventional DCSK due to elimination of one reference signal for each pair of bits.

\section{SYSTEM STRUCTURE}

The system is based on baseband implementation, perfect synchronization between transmitter and receiver is assumed

\section{A. Signal Format}

In DCSK system, each information bit will be sent by sending two similar chaotic sample functions having the same time duration. First time slot serves as a reference signal while the second time slot will be multiplied by the information signal. Hence, bit "1" is transmitted by sending the same chaotic sample function twice while " 0 " is transmitted by sending the reference signal followed by the its inverted copy. In the E-CDSK, the transmitted signal is divided in three parts as shown in Fig. 1.

- First part contains reference signal block $X_{M}$;

- Second part contains first $k_{t h}$ bit multiplied by the reference signal block, $b_{2 k} X_{M}$; where $\mathrm{k}$ is an integer .

- Third part contains next $(k+1)_{t h}$ bit multiplied by 
mirrored version of the reference signal block, $b_{2 k+1} X_{M}^{\prime}$.

\begin{tabular}{|c|c|c|c|c|c|}
\hline $\boldsymbol{X}_{\boldsymbol{M}}$ & $b_{o} \boldsymbol{X}_{\boldsymbol{M}}$ & $\boldsymbol{X}_{\boldsymbol{M}+1}$ & $b_{1} \boldsymbol{X}_{\boldsymbol{M}+1}$ & $\boldsymbol{X}_{M+2}$ & $b_{2} \boldsymbol{X}_{M+2}$ \\
\hline $\boldsymbol{X}_{\boldsymbol{M}}$ & $b_{o} \boldsymbol{X}_{\boldsymbol{M}}$ & $b_{I} \boldsymbol{X}_{M}^{\prime}$ & $\boldsymbol{X}_{M+1}$ & $b_{2} \boldsymbol{X}_{M+1}$ & $b_{3} \boldsymbol{X}_{M+1}^{\prime}$ \\
\hline
\end{tabular}

Fig. 1. Transmitted signal time slots for the conventional DCSK (upper part) and for the E-DCSK (lower part).

\section{B. Transmitter Description}

The transmitter generates a chaotic reference sequence $x_{i}$ of length $M$ as shown in Fig. 2. To transmit the first bit of information, the sequence will be followed by the same sequence multiplied by first bit $b_{2 k}$, The second information bit $b_{2 k+1}$ will be sent by multiplying the delayed and mirrored block of the original reference signal with $b_{2 k+1}$ and without sending another reference signal. Thus, the transmitted signal for any pair of bits $b_{2 k}$ and $b_{2 k+1}$ can be described as

$\begin{gathered}x_{i} \\ S_{i} \\ B\end{gathered}=\left\{\begin{array}{lr}4 k M<i \leq(4 k+1) M & (4 k+1) M<i \leq(4 k+2) M \\ b_{2 k}, x_{i-M} & (4 k+2) M<i \leq(4 k+3) M \\ 2 k+1 & x_{i-2 M}^{\prime}\end{array}\right\}$

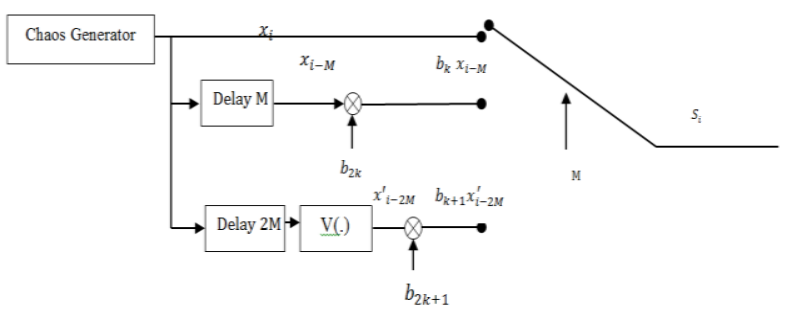

Fig. 2. Transmitter structure.

If the chaotic samples block is considered to be vector $\mathbf{X}$ with dimension $\mathbf{M}$ where $\mathbf{X}=\left(x_{1} x_{2} \ldots x_{M}\right)$. Then

$\mathbf{X}^{\prime}=\mathbf{V}(\mathbf{X})=P^{M} \mathbf{X}=\left(x_{1}^{\prime} x_{2}^{\prime} \ldots x_{M}^{\prime}\right)$.

where $P$ is a simple mapping matrix with of size $M \times M$ and it is defined as

$$
P=\left[\begin{array}{cccc}
0 & 0 & \cdots & 1 \\
0 & 0 & 1 & 0 \\
1 & 0 & \cdots & 0
\end{array}\right]
$$

Then

$$
\left(x_{1}^{\prime} x_{2}^{\prime} \ldots x_{M}^{\prime}\right)=\left(x_{M} x_{M-1} \ldots x_{1}\right)
$$

$x_{i_{-2 M}}^{\prime}$ represents the mapped block after delay of $2 M$.

For simplicity, we will keep using samples notation rather than matrix notation.

\section{Receiver}

Now, pair of information bits has been transmitted using single reference signal. Under the assumption that channel is Additive White Gaussian Noise, received signal sample $r_{i}$ can be written as:

$$
r_{i}=s_{i}+\psi_{i}
$$

where $\psi_{i}$ is a stationary random process with $E\left(\psi_{i}\right)=0$ and $\psi_{i}, \psi_{j}$ are statistically independent for any $\neq j$.

The receiver structure is shown in Fig. 3. In order to recover the first transmitted bit $b_{2 k}$, the received signal $r_{i}$ is multiplied by the received signal delayed by $M, r_{i-M}$. The second information bit is recovered by multiplying the received signal $r_{i}$ by the received signal delayed by $2 M$ and mapped by $V($.$) . Again.Recalling our definition for the$ mapped samples in (2)

$$
\left(r_{1}^{\prime}, r_{2}^{\prime}, \ldots r_{M}^{\prime}\right)=\left(r_{M}, r_{M-1}, \ldots r_{1}\right)
$$

Correlator outputs $Z_{1}$ and $Z_{2}$ can be formulated as:

$$
\begin{aligned}
& Z_{1}=\sum_{i=1}^{M} r_{i} r_{i-M} \\
& Z_{2}=\sum_{i=1}^{M} r_{i} r_{i-2 M}^{\prime}
\end{aligned}
$$

For the first correlator, when $(4 k+1) M<i \leq$ $(4 k+2) M$

The decision variable $Z_{1}$ can be written as:

$$
\begin{aligned}
& Z_{1}=\sum_{i=1}^{M}\left(s_{i}+\psi_{i}\right)\left(s_{i-M}+\psi_{i-M}\right) \\
& =\sum_{i=1}^{M}\left(b_{2 k} x_{i-M}+\psi_{i}\right)\left(x_{i-M}+\psi_{i-M}\right) \\
& =b_{2 k} \sum_{i=1}^{M} x_{i-M}^{2}+\sum_{i=1}^{M} \alpha_{i}
\end{aligned}
$$

For the second correlator, when $(4 k+2) M<i \leq(4 k+$ 3) $M$

The decision variable $Z_{2}$ can be written as:

$$
\begin{aligned}
& Z_{2}=\sum_{i=1}^{M}\left(s_{i}+\psi_{i}\right) V\left(s_{i-2 M}+\psi_{i-2 M}\right) \\
& Z_{2}=\sum_{i=1}^{M}\left(s_{i}+\psi_{i}\right)\left(s_{i-2 M}^{\prime}+\psi_{i-2 M}^{\prime}\right) \\
& =\sum_{i=1}^{M}\left(b_{2 k+1} x_{i-2 M}^{\prime}+\psi_{i}\right)\left(x_{i-2 M}^{\prime}+\psi_{i-2 M}^{\prime}\right) \\
& =b_{2 k+1} \sum_{i=1}^{M} x_{i-2 M}^{\prime 2}+\sum_{i=1}^{M} \beta_{i}
\end{aligned}
$$

where

$$
\begin{gathered}
\alpha_{i}=x_{i-M} \psi_{i}+b_{2 k} x_{i-M} \psi_{i-M}+\psi_{i} \psi_{i-M} \\
\beta_{i}=x_{i-2 M}^{\prime} \psi_{i}+b_{2 k+1} x_{i-2 M}^{\prime} \psi_{i-2 M}^{\prime}+\psi_{i} \psi_{i-2 M}^{\prime}
\end{gathered}
$$

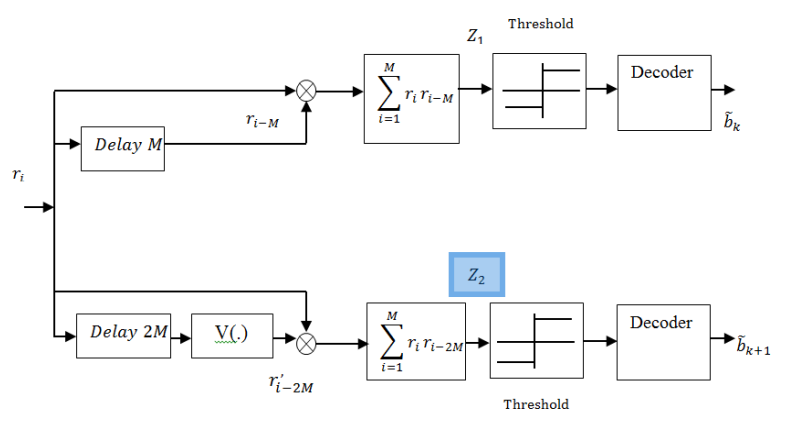

Fig. 3. Receiver structure.

The first two terms in (6) and (7) are the useful terms which contain the signal information, while the others terms $\alpha_{i}$ and $\beta_{i}$ represent the undesired signal components. The terms $\alpha_{i}$ and $\beta_{i}$ consist of noise terms and intrasignal interferenceterms which affects the information bearing signal part constructively and destructively [9]. Due to 
chaotic signal nature, each signal energy could be different form one bit to another [10], this also add another source of disturbance to the received signal. Generally both bits can be decoded according to the following equations

$$
\begin{aligned}
& \tilde{b}_{2 k} f\left(Z_{1}\right)= \begin{cases}-1, & Z_{1}<0 \\
+1, & Z_{1} \geq 0\end{cases} \\
& \tilde{b}_{2 k+1}=f\left(Z_{2}\right)= \begin{cases}-1, & Z_{2}<0 \\
+1, & Z_{2} \geq 0\end{cases}
\end{aligned}
$$

where $\tilde{b}_{2 k}$ and $\tilde{b}_{2 k+1}$ are the decoded bits.

\section{PERFORMANCE ANALYSIS}

To analyze the output for each correlator $Z_{1}$ and $Z_{2}$, the following assumptions are considered; $x_{i}$ is stationary and its distribution is uniform. Hence $\mathrm{E}\left(x_{i}\right)=0$ [6]. Correlation between chaotic samples $x_{k}$ and $x_{k+1}$ decay quickly. The same standard assumptions are also applied to $x_{i}^{\prime} . x_{i}$ and $\psi_{i}$ are statistically independent for all $i$ and $j$. For relatively large value of spreading factor, Gaussian approximation can be used to model the system which can be fully characterize by computing mean and variance for $Z_{1}$ and $Z_{2}$ respectively [11].

Under the aforementioned assumptions, it can be easily verified that the cross correlation of the three terms in equations (8) and (9) have an average value of zero [9] .The correlator output $Z_{1}$ in equation (4) can be rewritten as:

$$
Z_{1}=b_{2 k} A+b_{2 k} \eta+\alpha
$$

Two information bits will be sent using only three chaotic sample functions, thus $A=\sum_{i=1}^{M} E\left(x_{i}{ }^{2}\right), A=M . \sigma_{x}^{2}=2 / 3 E_{b}$ 。 where $E$ (.) is a mathematical expectation function and $E_{b}$ is average bit energy for the transmitted signal.

The factor $\eta$ depends on the chaotic system which generates the chaotic samples and it is given as $\eta=\sum_{i=1}^{M} x_{i}^{2}-A$

In our system, Tent Map is chosen to generate the chaotic sequences which have uniform distribution and it is given by

$$
x_{n+1}=1-1.99\left|x_{n}\right|
$$

Thus, $\sigma_{\eta}^{2}$ can be found [9]

$$
\sigma_{\eta}^{2}=\left(2 * \frac{2}{3}\right)^{2} \frac{E_{b}^{2}}{5 M}=\frac{16 E_{b}^{2}}{45 M}
$$

Now calculating the $\sigma_{\alpha}^{2}$, which is equal to the variance of each component in eq. (8)

$$
\begin{aligned}
\sigma_{\alpha}^{2}= & \sum_{i=1}^{M} \sigma_{x}^{2} \sigma_{o}^{2}+\sum_{i=1}^{M} \sigma_{x}^{2} \sigma_{o}^{2}+\sum_{i=1}^{M} \sigma_{o}^{2} \sigma_{o}^{2}=\frac{2}{3} E_{b} \sigma_{o}^{2}+ \\
& \frac{2}{3}+E_{b} \sigma_{o}^{2}+M . \sigma_{o}^{4}
\end{aligned}
$$

We will define $\sigma_{o}^{2}=\frac{N_{o}}{2}$, where $N_{o}$ is the noise spectral power density in the channel, then

$$
\sigma_{\alpha}^{2}=\frac{4}{3} E_{b} \cdot \frac{N_{o}}{2}+M \cdot \frac{N_{o}}{2} \cdot \frac{N_{o}}{2}=\frac{4}{3} E_{b} \cdot N_{o}+\mathrm{M} \cdot\left(\frac{N_{o}^{2}}{4}\right)
$$

Therefore

$$
\sigma_{\alpha+\eta}^{2}=\frac{4}{3} E_{b} \cdot N_{o}+\mathrm{M} \cdot\left(\frac{N_{o}^{2}}{4}\right)+\frac{16 E_{b}^{2}}{45 M}
$$

In the same manner $\sigma_{\alpha+\eta}^{2}$ can be derived for $Z_{2}$

Since our assumption that the channel is Gaussian, BER can be given by

$$
\mathrm{BER}=\frac{1}{2} \operatorname{erfc}\left(\frac{A}{\sqrt{2 . \sigma_{\alpha+\eta}^{2}}}\right)
$$

where $\operatorname{erfc}(x)=\frac{2}{\sqrt{\pi}} \int_{x}^{\infty} e^{-t^{2}} d t$

Thus, equation can be rewritten as

$$
B E R_{E-D C S K}=\frac{1}{2} \operatorname{erf} c\left(\sqrt{\frac{4 E_{b}}{9 N_{o}}\left(\frac{4}{3} \frac{E_{b}}{N_{o}}+\frac{M}{2} \frac{N_{o}}{E_{b}}+\frac{32}{45} \frac{E_{b}}{N_{o}}\right)^{-1}}\right)
$$

\section{Simulation}
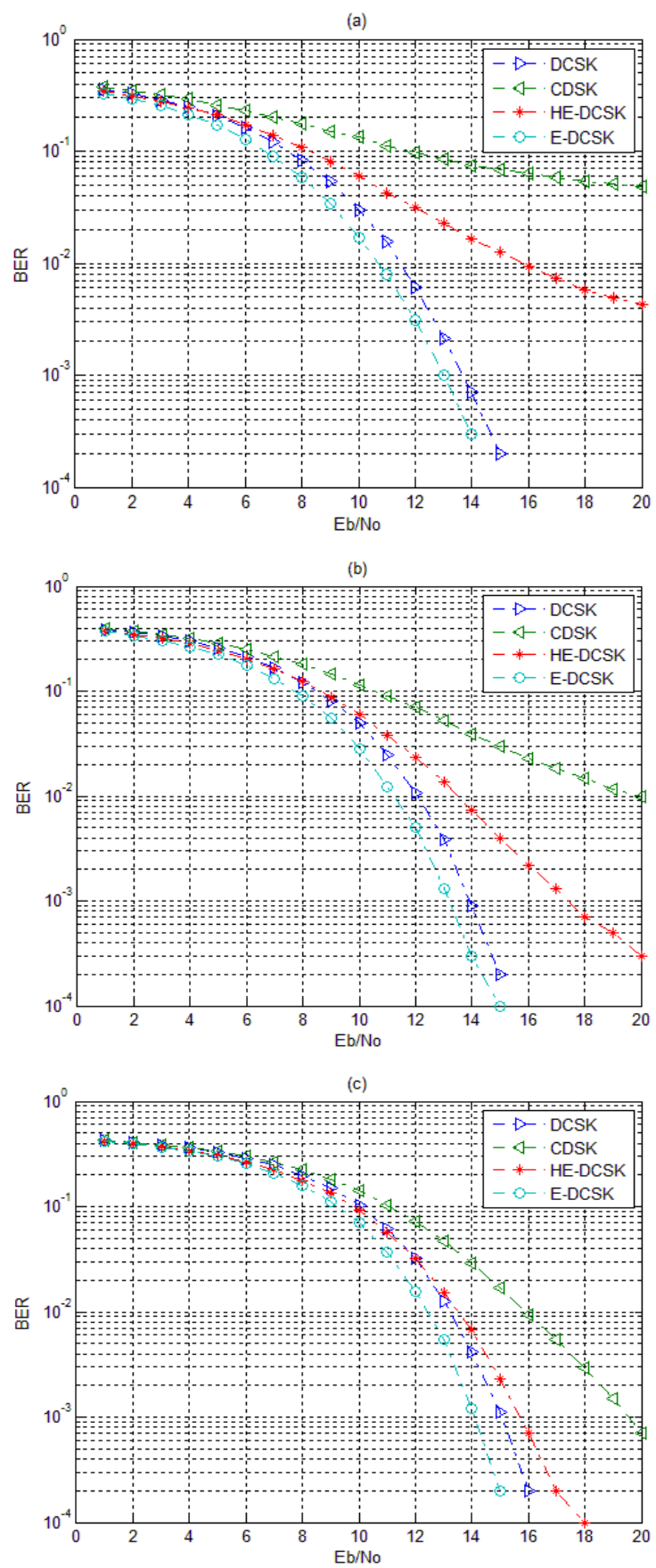


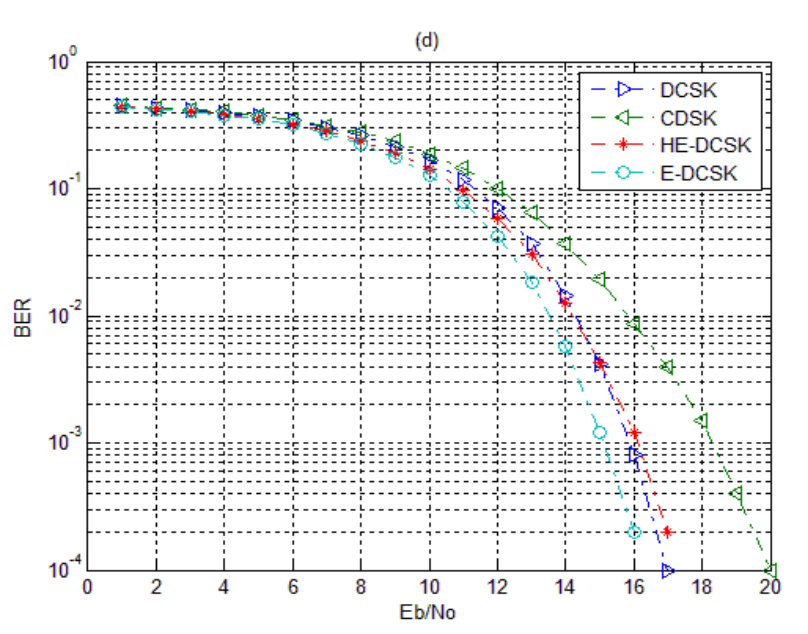

Fig. 4. BER performances of DCSK, CDSK, HE-DCSK and E-CDSK in AWGN channel with various spreading factor (a) $M=10$. (b) $M=20$. (c) $M=50$. (d) $M=100$.

In this section, different chaos based communications systems, (CDSK, CDSK, and HE-DCSK and E-DCSK, are simulated under AWGN channel environment and with different spreading factors $M$. Also, theoretical performance results for the proposed system are compared with the simulation results at $M=100$.

Obviously, in Fig. 4(a) with spreading factor $M=10$ and at low SNR, E-DCSK seems always $1 \mathrm{~dB}$ better than HE-DCSK and DCSK; and $2 \mathrm{~dB}$ better than CDSK. The reason of which is that there are much more intrasignal interference signals components in the correlator output of HE-DCSK and CDSK Clear improvement can be noticed at higher $E_{b} / N_{\mathrm{o}}$ levels. When $M=20$ and $M=50$ as shown in Fig. 4(a) and Fig. 4(b), E-DCSK shows same performance if it is compared with other chaos based communication systems at lower $E_{b} / N_{\mathrm{o}}$ while E-DCSK can gain an average of $1 \mathrm{~dB}$ with respect to other systems at higher levels of $E_{b} / N_{\mathrm{o}}$. When $M=100$, it is interesting to note that the proposed system has equal noise performance compared with other system due to the increase of the noise interference components in the correlator output, the effect of noise will be degraded at high levels of $E_{b} / N_{\mathrm{o}}$ and the proposed system still outperforms the other systems.

Clear matching between the theoretical term for BER of the proposed system and the simulation result at $M=100$ in Fig. 5. This validate our equation (11) which is based on Gaussian approximation method

\section{CONCLUSION}

A new scheme which is based on extension of conventional DCSK is developed. Using the same reference signal for each different pair of bits, the proposed system reduces bandwidth required for transmission and the average transmitted bit energy for transmission. Bit error performance for the presented system is simulated and compared with other chaos based communication systems. The system shows better performance than other chaos based communication system due to reduction in the intrasignal interference terms that affects the overall performance at low spreading factors.

Simulations under AWGN channel show that the E-CDSK overtakes the other chaos based communication schemes by an average of 1-2 $\mathrm{dB}$ at low spreading factors. Clear matching is obtained between the BER analytical expressions with simulation results.

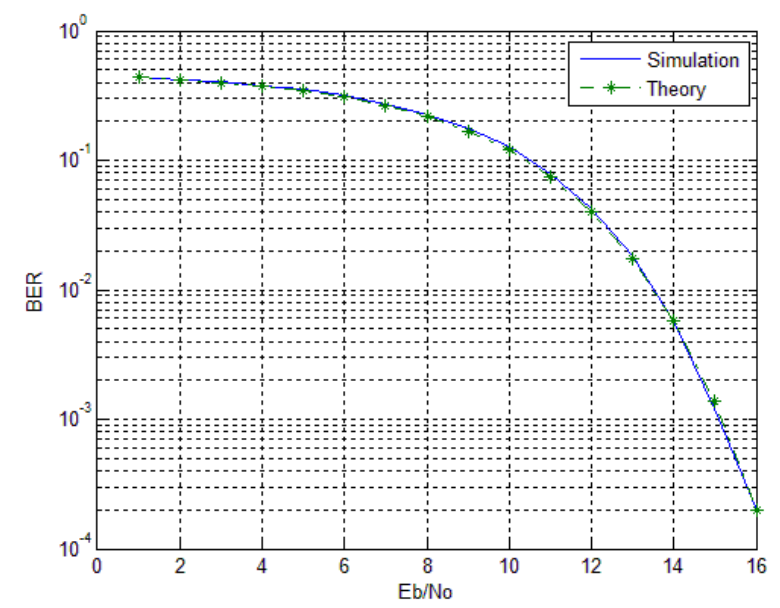

Fig. 5. Relationship between theoretical BER performance and $E b / N_{\mathrm{o}}$ for E-DCSK systems with spreading factor $M=100$.

\section{REFERENCES}

[1] G. Kolumbán, M. P. Kennedy, and L. O. Chua, "The Role of Synchronization in Digital Communications Using Chaos- II: Chaotic Modulation and Chaotic Synchronization," IEEE Trans. Circuits Syst.I: Fundamental Theory and Applications, vol. 45, no. 11, pp. 1129-1140, Nov. 1998.

[1] W. M. Tam, F. C. Lau, and C. K. Tse, "Digital Communications with Chaos Multiple Access Techniques and Performance," Elsevier Science, 2006, pp. 12-31.

[2] G. Kolumbán, Z. Jákó, and M. P. Kennedy, "Enhanced Versions of DCSK and FM-DCSK data transmissions systems," in Proc. IEEE ISCAS'99, vol. 4, Orlando, FL, 1999, pp. 475-478.

[3] K. Y. Cheong, F. C. Lau, and C. K. Tse, "Permutation- Based M-ary Chaotic-Sequence Spread Spectrum Communications Systems," Circuits, Systems Signal Processing, vol. 22, no. 6, pp. 567-577, Jan. 2003.

[4] F. C. Lau, K. Y. Cheong, and C. K. Tse, "Permutation-Based DCSK and Multiple-Access DCSK Systems," IEEE Trans. Circuits Syst.I: Fundamental Theory and Applications, vol. 50, no. 6, pp. 733-742, June 2003.

[5] H. Yang and G. Jiang, "High-Efficiency Differential-Chaos-Shift-Keying Scheme for Chaos-Based Noncoherent Communication," IEEE Trans. Circuits Syst.II: Express Briefs, vol. 59, no. 5, pp. 312-316, May 2012

[6] G. Kaddoum and F. Gagnon, "Design of a High-Data-Rate Differential Chaos-Shift Keying System," IEEE Trans. Circuits Syst,II: Express Briefs, vol. 59, no. 7, pp. 448-452, July 2012.

[7] M. Sushchik, L. S. Tsimring, and A. R. Volkovskii, "Performance Analysis of Correlation-Based Communication Schemes Utilizing Chaos," IEEE Transactions on Circuits And Systems - I: Fundamental Theory And Applications, vol. 47, no. 12, pp. 1684-1691, 2000.

[8] G. Kaddoum, P. Chargé, and D. Roviras, "A Generalized Methodology for Bit-Error-Rate Prediction in Correlation-Based Communication Schemes Using Chaos," IEEE Communications Letters, vol. 13, no. 8, pp. 567-569, 2009.

[9] A. Abel, M. Gotz, and W. Schwarz, "Statistical Analysis of Chaotic Communication Schemes," IEEE International Symposium on Circuits and Systems( ISCAS '98), Monterey, 1998, pp. 465-468.

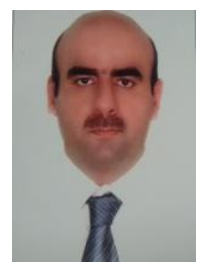

Nizar N. Albasamwas was born in Baghdad, Iraq in 1975. He received the M.Sc. degree from Al-Mustansyria University, Baghdad, Iraq in 2000 and $\mathrm{Ph} . \mathrm{D}$. degree form AL-Nahrain University, Baghdad, Iraq in 2004, Currently $\mathrm{He}$ is working as a faculty member in the department of Electronics and Communication in Middle East College, Muscat, Oman. His primary research interests are spread spectrum systems and chaos communications. 